\title{
DIABETES MELLITUS IN PARKINSON'S DISEASE PATIENTS
}

\author{
Borislav Ivanov, Ara Kaprelyan, Margarita Grudkova, Iliya Dimitrov, Nadezhda Deleva \\ First Clinic of Neurology, St. Marina University Hospital of Varna
}

\begin{abstract}
PURPOSE: Parkinson's disease (PD) is the second most common neurodegenerative disease affecting approximately 5 million people worldwide. Some studies have found out an association between PD and type 2 diabetes mellitus (DMT2). Their results suggest a common pathophysiologic pathway. Other authors fail to establish any link between these two diseases. The aim of the present study is to search for a potential association between DMT2 and PD.

MATERIAL AND METHODS: We investigated the medical records of 85 patients, 45 males and 40 females, aged 68,02 $\pm 9,02$ (range, 40-87) years and with disease duration of 4,98 $\pm 3,96$ (range, 1-18) years. They were diagnosed as PD according to the United Kingdom Parkinson's Disease Society Brain Bank Diagnostic Criteria and hospitalized for a two-year period.

RESULTS: We established a prevalence of DMT2 in PD patients of $18,8 \%$. We discussed the potential relationships between these two disorders of great medical, social and economic impact. Insulin levels contribute to normal brain function.
\end{abstract}

CONCLUSION: The history of DMT2 or the use of antidiabetic drugs might be a risk factor for PD.

Key words: Parkinson's disease, type 2 diabetes mellitus, diagnosis, risk factor

\section{INTRODUCTION}

Parkinson's disease (PD) is the second most common neurodegenerative disease that affects approximately 5 million people worldwide. Its etiology is poorly understood; however, vascular factors may be influential in modulating the disease risk (13).

Besides PD may be related to mitochondrial dysfunction, oxidative stress, excitotoxicity, apoptosis, and inflammation. Chronic systemic inflammation

Address for correspondence:

Borislav Ivanov, $M D, P h D$

First Clinic of Neurology,

St. Marina University Hospital of Varna

1 Hristo Smirneneski Str., 9010 Varna, Bulgaria

E-mail:borislav.ivanov@mail.bg

Phone: +359-52978545

Received: August 30, 2012

Accepted: January 15, 2013 or impaired mitochondrial metabolism have been suspected in the development of diabetes mellitus (DM), too (2).

Type 2 DM (DMT2) may lead to PD through suppression of central dopamine levels and cerebrovascular disease. PD development may influence on DM risk, too. Dopaminergic neurons participate in feeding behaviour in low glucose levels. This feedback loop is mediated by insulin receptors in the substantia nigra. Dopamine agonists such as bromocriptine affect the peripheral glucose control. Changes in glucose control associated with loss of dopaminergic function might occur early in the course of PD, perhaps even before motor symptoms develop (5).

Some studies have found out an association between PD and DM. Their results suggest a common pathophysiologic pathway and DM or antidiabetictreatment predicted PD risk $(6,12,14)$. Other authors 
fail, however, to establish any link between these two diseases $(5,10)$.

The aim of this study was to search for a potential association between PD and DMT2.

\section{MATERIAL AND METHODS}

For a two-year period, 85 PD patients were hospitalized in the First Clinic of Neurology, St. Marina University Hospital of Varna. They were diagnosed according to the United Kingdom Parkinson's Disease Society Brain Bank Diagnostic Criteria. The patients were 45 males and 40 females, aged 68,02 $\pm 9,02$ (range, 40-87) years and with disease duration of 4,98 $\pm 3,96$ (range, 1-18) years. We examined the medical records of these patients for history or clinical data about DMT2 and insulin or oral antidiabetic treatment.

\section{RESULTS}

We found out $16 \mathrm{PD}$ patients $(18,8 \%$ of the cases) presenting with DMT2. They were 6 males and 10 females, aged 69,88 $\pm 10,99$ (range, 54- 87) years and with disease duration of $4,75 \pm 4,42$ (1-15) years. DMT2 preceded PD in 10 cases.

Six out of 16 patients were on insulin treatment (4 in the group preceding the debut of PD), one patient was on oral antidiabetic drugs and the remaining 9 patients were on diet only.

\section{DISCUSSION}

Our results show a prevalence of DMT2 in PD patients of $18,8 \%$. This rate is similar to that reported in the age-matched general population (prevalence of $19,3 \%$ for the age over 59 years) (3).

There are controversial opinions concerning the relationships between PD and DMT2. Insulin and glucose levels contribute to the normal brain function. The history of DMT2 or the use of antidiabetic drugs might be a risk factor for PD. One study has demonstrated that DMT2 is associated with a $36 \%$ increased risk of developing $\mathrm{PD}(4,8,11,12)$.

Other authors state that neither history of DMT2, nor body mass index is significantly related with PD risk $(5,10)$.
One study reveals a significant up-regulation of substantia nigra genes in PD which possess wellknown biological associations with cancer, $\mathrm{DM}$, and inflammation (10).

Previous observational studies have reported inconsistent results concerning the association between DM and PD, and data about the risk of developing incident $\mathrm{DM}$ in relation to $\mathrm{PD}$ are scarce. In another observational study, DM prevalence is closely similar between patients with PD and subjects without this disorder. The risk of developing incident $\mathrm{DM}$ is lower for patients with PD than for subjects without PD.

PD risk is not significantly related to history of hypertension, hypercholesterolemia, or DM (13).

There are inconsistent results concerning the association between DM and PD comparing the DM prevalence between patients with or without PD and at exploring the risk of developing incident $\mathrm{DM}$ associated with PD. The risk of developing an incident DM tends to be lower in PD patients than in subjects without PD (2). Obviously, DM is not a preceding risk factor for $\mathrm{PD}(5)$.

Our results support the observations of the lack of association between DMT2 and PD. Most probably, DMT2 is linked to secondary Parkinsonism with vascular and drug-induced risk factors.

\section{REFERENCES}

1. Arvanitakis, Z., R. Wilson, J. Bienias, D. Bennett. Diabetes and parkinsonian signs in older persons.Alzheimer Dis. Assoc. Disord., 21, 2007, No 2, 144-149.

2. Becker, C., G. Brobert, S. Johansson, S. Jick, C. Meier. Diabetes in patients with idiopathic Parkinson's disease.- Diabetes Care, 31, 2008, No 9, 1808-1812.

3. Bringer, J., P. Fontaine, B. Detournay, F. NachitOuinekh, G. Brami, E. Eschwege. Prevalence of diagnosed type 2 diabetes mellitus in the French general population: the INSTANT study.- Diabetes Metab., 35, 2009, No 1, 25-31.

4. D’Amelio, M., P. Ragonese, G. Callari, N. Di Benedetto, B. Palmeri, V. Terruso, et al. Diabetes preceding Parkinson's disease onset. A casecontrol study.- Parkinsonism Relat. Disord., 15, 2009, No 9, 660-664. 
5. Driver, J., A. Smith, J. Buring, J. Gaziano, T. Kurth, G. Logroscino. Prospective cohort study of type 2 diabetes and the risk of Parkinson's disease.Diabetes Care, 31, 2008, No 10, 2003-2005.

6. Jagota, P., R. Bhidayasiri, A. Lang. Movement disorders in patients with diabetes mellitus.- $J$. Neurol. Sci., 314, 2012, No 1-2, 5-11.

7. Ma, H., J. Kim, M. Chu, M. Oh, K. Yu, J. Kim, W. Hahm, Y. Kim, B. Lee. Diabetes mellitus and drug-induced Parkinsonism: a case-control study.J. Neurol. Sci., 284, 2009, No 1-2, 140-143.

8. Miyake, Y., K. Tanaka, W. Fukushima, S. Sasaki, C. Kiyohara, Y. Tsuboi, et al.; Fukuoka Kinki Parkinson's Disease Study Group. Case-control study of risk of Parkinson's disease in relation to hypertension, hypercholesterolemia, and diabetes in Japan.- J. Neurol. Sci., 293, 2010, No 1-2, 82-86.

9. Moran, L., M. Graeber. Towards a pathway definition of Parkinson's disease: a complex disorder with links to cancer, diabetes and inflammation.- Neurogenetics, 9, 2008, No 1, 1-13.
10. Palacios, N., X. Gao, M. McCullough, E. Jacobs, A. Patel, T. Mayo, M. Schwarzschild, A. Ascherio. Obesity, diabetes, and risk of Parkinson's disease.Mov. Disord., 26, 2011, No 12, 2253-2259.

11. Riederer, P., J. Bartl, G. Laux, E. Grünblatt. Diabetes type II: a risk factor for depressionParkinson-Alzheimer?- Neurotox. Res., 19, 2011, No 2, 253-265.

12. Schernhammer, E., J. Hansen, K. Rugbjerg, L. Wermuth, B. Ritz. Diabetes and the risk of developing Parkinson's disease in Denmark.Diabetes Care, 34, 2011, No 5, 1102-1108.

13. Simon, K., H. Chen, S. Michael, A. Ascherio. Hypertension, hypercholesterolemia, diabetes, and risk of Parkinson disease.- Neurology, 69, 2007, No 17, 1688-1695.

14. Xu, Q., Y. Park, X. Huang, A. Hollenbeck, A. Blair, A. Schatzkin, H. Chen. Diabetes and risk of Parkinson's disease.- Diabetes Care, 34, 2011, No 4, 910-915. 